\title{
Detailed elevation map of Ice Stream C, Antarctica, using satellite imagery and airborne radar
}

\author{
R. A. BINDSGHADLER \\ Code 971, NASA/Goddard Space Flight Center, Greenbell, MD 20771, U.S.A. \\ P. L. VORNBERGER \\ General Sciences Corporation, Laurel, MD 20707-2929, U.S.A.
}

\begin{abstract}
Surface elevations collected by airborne radar are interpolated between flight lines using Landsat TM image data to produce a detailed digital elevation model of a part of Ice Stream C, West Antarctica. The photoclinometric method developed is general and allows the derivation of surface elevation along any line parallel to the solar illumination from a single known elevation on that line. Accuracies of the derived elevations are improved with additional clevation control that permits an empirical determination of parameters in the photoclinometric equation accounting for albedo and atmospheric scattering. Elevation errors increase approximately linearly with the integration distance. An explicit relationship is derived that shows the image brightness is insensitive to the cross-Sun component of small surface slopes typical of ice sheets. The greatest impediment to the accuracy of this technique is the radiance resolution of the sensor which limits the signal-to-noise ratio of the image data and can mask variations in albedo that arc falsely converted to variations in surface slope.
\end{abstract}

\section{INTRODUCTION}

Because of snow's homogeneous reflectivity, appropriately enhanced, high-resolution satellite imagery shows the topographic details of the ice-sheet surface (Dowdeswell and McIntyre, 1987; Rees and Dowdeswell, 1988; Vaughan and others, 1988). Quantitative extraction of this information has value in a number of applications including flow-dynamics studies and field operations. Driving stresses depend on the geometric parameters of surface slope and ice thickness. Proportionally, surface slope varies much more than ice thickness and thus is responsible for most of the spatial variation in driving stress (Cooper and others, 1982). Detailed knowledge of surface elevation allows a more precise determination of this driving stress as required by more sophisticated iceflow models.

Newer, more accurate methods of measuring surface elevation, such as airborne laser altimetry and surfacekinematic GPS, have been developed in recent ycars but, even with these advanced systems, the collected data are highly linearized with large gaps between the measured lines. Satellite imagery can be uscd spatially to interpolate linearized elevation data, thus generating a complete digital-clevation model (DEM), for which an elevation value exists at every image pixel. In this paper, we develop this approach and demonstrate its feasibility.

\section{PHOTOCLINOMETRY}

The technique of extracting topographic information from image data is called "photoclinometry" and is well established in planetary science (Wildey, 1975). The photometric equation relates the sensor-measured radiance to the illumination of the surface, the reflecting properties of that surface and the geometric configuration of the sensor, Sun and surface. Measurements of the bidirectional reflectance function of snow support the simplifying assumption of a cosine reflector with a constant surface reflectivity when viewed at nadir (Steffen, 1987). The photometric equation can then be written as

$$
\mathrm{DN}=C\left[I R(\mathbf{S} \cdot \mathbf{n})-L_{0}+T\right]
$$

where DN is the usual "digital number" expressing image brightness, $I$ is the incident solar radiance, $R$ is the reflectivity of the surface, $C$ is the conversion from radiance to sensor DN units, $S$ is the unit vector pointing from the surface toward the Sun, $\mathbf{n}$ is the surface-normal unit vector, $L_{0}$ is the minimum radiance threshold of the sensor and $T$ represents all other radiancc sources, such as atmospheric scattering. The dot product

$$
\mathbf{S} \cdot \mathbf{n}=\cos \theta
$$




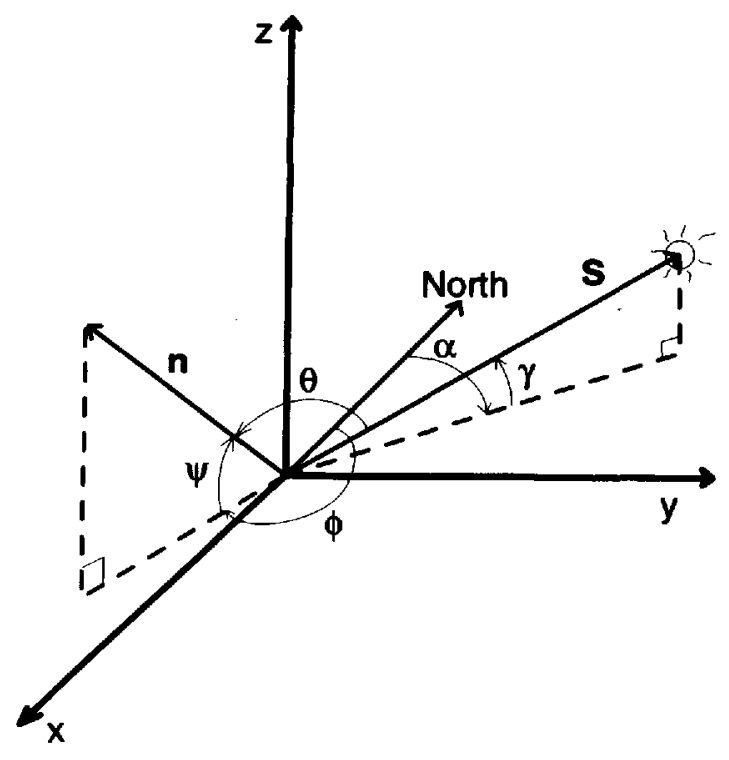

Fig. 1. Generalized geometry for photoclinometry. Definilions of the angles are given in the text.

where $\theta$ is the illumination angle (see Fig. 1). $\mathbf{S}$ and $\mathbf{n}$ are described in a general, righthanded coordinate with a zenith-pointing $z$ axis as:

$$
\mathbf{S}=a \mathbf{i}+b \mathbf{j}+c \mathbf{k}, \quad a^{2}+b^{2}+c^{2}=1
$$

and

$$
\mathbf{n}=d \mathbf{i}+e \mathbf{j}+f \mathbf{k}, \quad d^{2}+e^{2}+f^{2}=1 .
$$

The coefficicnts of the vectors are related to the azimuth and clevation angles of the Sun and surface normal. As discussed below, selection of the particular coordinatc system, where one axis is aligned with the Sun's azimuth, provides unique advantages. In this case,

$$
\begin{array}{lll}
a=\cos \gamma & b=0 & c=\sin \gamma \\
d=\cos (\varphi-\alpha) \cos \psi & c=\sin (\varphi-\alpha) \cos \psi & f=\sin \psi(6)
\end{array}
$$

where $\alpha$ and $\gamma$ arc the respective azimuth and elevation angles of the Sun, and $\varphi$ and $\psi$ are the respective azimuth and elevation angles of the surface-normal vector.

In the Sun-aligned coordinate system, the total surface slope has two components:

$$
\begin{aligned}
& \text { along-Sun component, } \quad \alpha_{\mathrm{a}}=\tan ^{-1}(d / f), \text { and } \\
& \text { cross-Sun component, } \quad \alpha_{\mathrm{c}}=\tan ^{-1}(e / f) .
\end{aligned}
$$

From Equations (2) through (8), the illumination angle can be written as

$$
\cos \theta=\frac{\tan \alpha_{\mathrm{a}} \cos \gamma+\sin \gamma}{\operatorname{sqr}\left(\tan ^{2} \alpha_{\mathrm{a}}+\tan ^{2} \alpha_{\mathrm{c}}+1\right)} .
$$

Equation (9) shows that, although $\cos \theta$ (and, by Equation (1), the image brightness) depends on both components of the total slope, the sensitivity of $\cos \theta$ to $\alpha_{a}$ and $\alpha_{\mathrm{c}}$ differs considerably. This has been noted before (e.g. Rees and Dowdeswell, 1988) but Equation (9) gives the explicit relationship. The Sun-aligned coordinate system optimizes the separation of the total slope into components that are most sensitive and least sensitive to the image brightness. This effect can be quantified as the ratio of the partial derivatives of $\mathrm{DN}$ with respect to $\alpha_{\mathrm{a}}$ and $\alpha_{\mathrm{c}}$,

$$
\frac{\partial(\mathrm{DN}) / \partial \alpha_{\mathrm{a}}}{\partial\left(\mathrm{DN} / \partial \alpha_{\mathrm{c}}\right.}=\frac{\cos ^{2} \alpha_{\mathrm{c}}}{\cos ^{2} \alpha_{\mathrm{a}}} \frac{\tan \alpha_{\mathrm{a}} \tan \gamma-\tan ^{2} \alpha_{\mathrm{c}}-1}{\tan \alpha_{\mathrm{c}}\left(\tan \gamma+\tan \alpha_{\mathrm{a}}\right)} \text {. }
$$

This ratio is large for small slopes when $\gamma<45^{\circ}$. Another way of stating this result is that the characteristics of $\operatorname{DN}\left(\alpha_{\mathrm{i}}, \alpha_{\mathrm{c}}\right)$ lie nearly parallel to the $\alpha_{\mathrm{c}}$ axis. The singularity in Equation (10) at $\tan \alpha_{\mathrm{c}}=0$ is a result of $\alpha_{\mathrm{c}}$ having no effect on image brightness for that particular geometry. The other singularity, $\tan \gamma=\tan \alpha_{\mathrm{c}}$, corresponds to a condition of grazing incidence, at which point shadows will begin to occur and the photoclinometric equation is invalid. For the specific case considered in this paper, $\gamma=16^{\circ}$ and the pixel-scale surface slopes have a $3 \sigma$ range of $\pm 0.007 \mathrm{rad}$, so the ratio of sensitivities in Equation $(10)$ is above 485 in $99 \%$ of the region. The significance of this separation is that an accurate value of $\alpha_{\mathrm{a}}$ can be calculated from $\cos \theta$ using Equation (9) without knowing $\alpha_{c}$, as Iong as the small-slope criterion is satisfied.

Returning to the photoclinometric equation, Equations (1) and (2) are combined as

$$
\mathrm{DN}=A \cos \theta+B
$$

where

$$
\begin{aligned}
& A=C I R \text { and } \\
& B=C\left(-L_{0}+T\right) .
\end{aligned}
$$

Once $A$ and $B$ are specificd, Equation (11) can be used to convert DN to illumination angle. Next, Equation (9) is used to estimate $\alpha_{\mathrm{a}}$ (assuming $\alpha_{\mathrm{c}}=0$, for convenience). These slopes can then be integrated along a Sun-parallel line from an initial, known elevation value on that line to generate a continuous elevation profile one-pixel wide. These independent elevation profiles are related by the set of known elevations that are used to begin the integration. It is through this set of known elevations, in our case the initial up-Sun radar flightline, that information on the cross-Sun slope is incorporated into the DEM. However, due to the insensitivity of $\cos \theta$ on the cross-Sun slopes, very large values of cross-Sun slope can gradually evolve between adjacent along-Sun profiles for even modest noise in the DN values, particularly for longer integration distances. These large slopes are belicved to be artifacts, because they fall well outside the distribution of small-scale surface slopes either measured by airborne radar or calculated in the along-Sun direction. To control this effect, a crossSun smoothing scheme, using a running average, was applied to cach cross-Sun profile during the along-Sun integration process. Running averages of different lengths were attempted. Unrealistically large cross-Sun slopes (i.c. slopes well outside the expected distribution) remained for filters shorter than 31 pixels $(883.5 \mathrm{~m})$. Thus, a 31 pixel-wide filter was used. This smoothing 
eliminated the cross-Sun artifacts without noticeably disturbing the along-Sun integration.

\section{DATA}

The opportunity to test this technique arose when personnel from the University of Wisconsin collected detailed surface-elcvation data of a region of Ice Stream C. West Antarctica, using an airborne-radar system Retzlaff and others, 1993). Data of surface elevation were spaced approximately every $140 \mathrm{~m}$ along flight lines spaced about $5 \mathrm{~km}$ apart. A detailed error analysis of this data set yielded a calculated $1 \sigma$ crror of $\pm 3.7 \mathrm{~m}$ for surface elevation (Retzlaff and others, 1993).

This area also falls within the coverage of Landsat, and we had in our possession a Thematic Mapper (TM) image of this area collected on 24 January 1985 (ID\#: Y5032913355X0; path 226, row 121). Band 4 was chosen as the single band with the best representation of surface features. Figure 2 shows the band 4 image (quad 2) of this area and the position of the radar flight lines. The sizc of this sub-image is $91.9 \mathrm{~km} \times 84 \mathrm{~km}$ with a pixel resolution of $28.5 \mathrm{~m}$ and the center of the scene is located at $82.0581^{\circ} \mathrm{S}, 135.0544^{\circ} \mathrm{W}$. The Sun elevation at the center of the image shown is $15.79^{\circ}$ at an azimuth of $117.3^{\circ}$ from true north and was calculated from the location of the image and Greenwich Mean Time of acquisition.

From the airborne-radar data alone, Retzlaff and others (1993) produced a contour map of surface elevation by computer interpolation. By a similar method employing kriging principles, we produced a contour map nearly identical to the Retzlaff and others' map. Figure 3 shows this contour map superimposed on the Landsat image and a shaded relief version of the map. Comparison of the shaded relief map, with the actual TM image, shows that while the contour map does indicate properly the existence of, and position of, many major topographic features, it misrepresents major features not positioned near a flight line and poorly represents smaller topographic undulations between flight lines. Additionally, many of the more subtle features such as the flow traces, that are continuous over large distances, were missing from the contour map. Different values of interpolation parameters did not eliminate these differences.

Before our photoclinometric method was applied to the image, a number of processing stcps were taken. Image scan-line noise was removed by standard image-

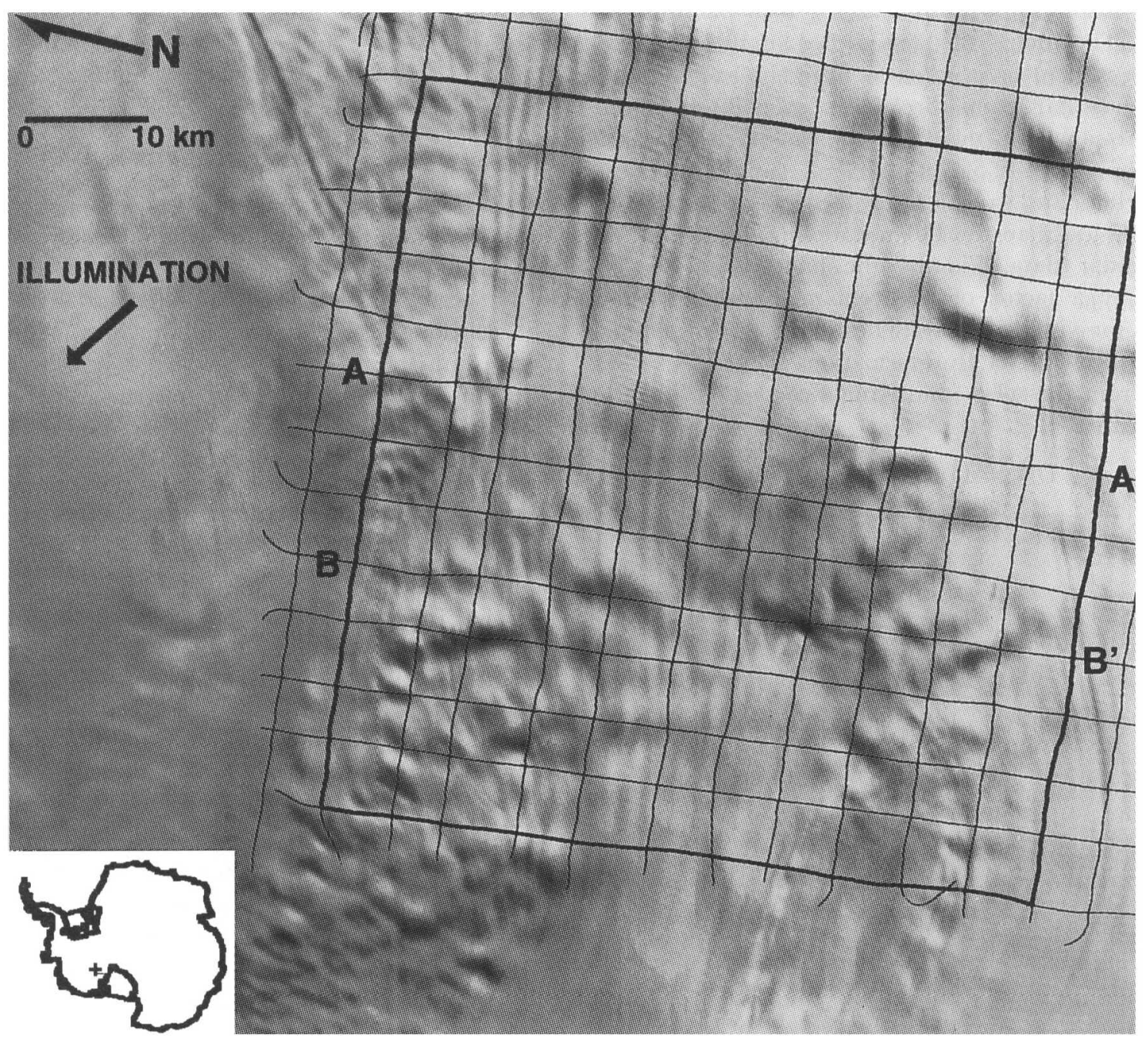

Fig. 2. Landsat TM image of study area with airborne flight lines superimposed. Heavy lines indicate the box over which the photoclinometric method was applied. The inset shows geographic position of area on Ice Stream C. Profiles $A-A^{\prime}$ and $B-B^{\prime}$ are shown in Figure 8. 

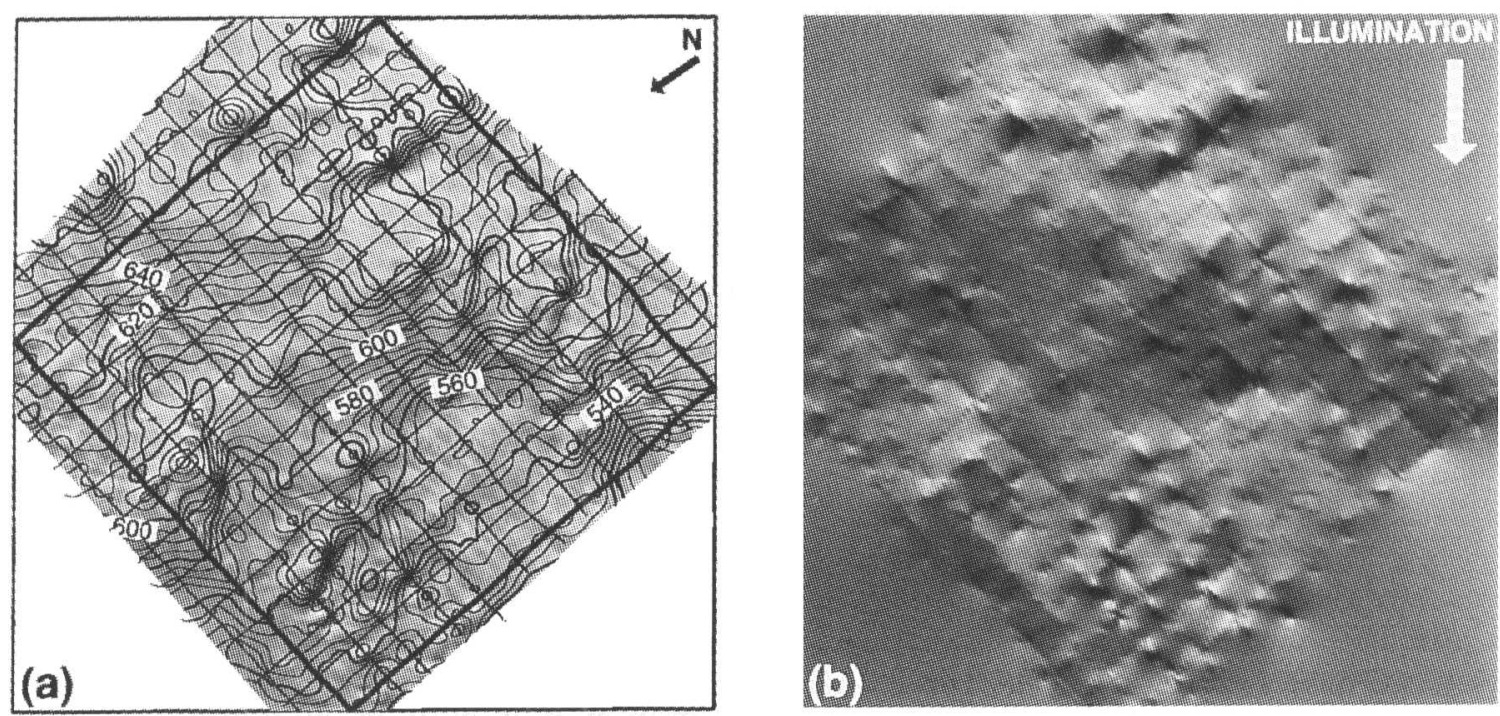

Fig. 3. a. Elevation-contour map based on airborne-radar data only. Contour interval is $5 \mathrm{~m}$. Contours are superimposed on original image. $b$. Shaded relief version of contours in (a) illuminated from the direction indicated. This illumination direction corresponds to the direction in the original image. These images, and those of Figures 5, 6 and 9, are displayed in the Sun-aligned coordinale system described in the text.

processing techniques (Crippen, 1989). Then, each pixel DN value was divided by the cosine of the solar zenith angle for that pixel to normalize the solar-illumination gcomctry. The image was re-sampled in the preferred coordinate system, i.e. rotated to align the axes parallel and perpendicular to the direction of solar illumination. After the re-sampling, the data were smoothed using a 5 pixel $\times 5$ pixel $(142.5 \mathrm{~m} \times 142.5 \mathrm{~m})$ running-average filter to climinate variation in the image data at a spatial scale less than the $140 \mathrm{~m}$ sampling interval of the airborne-radar data. Finally, the image was linearly stretched by a factor of 3 to enhance the brightness variations for visual display. The mathematical expression that represents the transformations to the $D N$ values resulting from the solar zenith-angle correction and the enhancement is

$$
\mathrm{DN}^{\prime}=11.025 \mathrm{DN}-890
$$

where $\mathrm{DN}$ and $\mathrm{DN}^{\prime}$ are the original and enhanced $\mathrm{DN}$ values, respectively.

\section{APPLICATION}

One method to obtain values for $A$ and $B$ in Equations (12) and (13) is to use sensor and environmental characteristics. TM Band 4 spans the spectral range $760-900 \mathrm{~nm}$. In this part of the spectrum,

$$
\begin{aligned}
C & =12.28 \mathrm{~cm}^{2} \mu \mathrm{m} \mathrm{mW}^{-1}, \\
I & =33.33 \mathrm{~mW} \mathrm{~cm}^{-2} \mu \mathrm{m}^{-1}
\end{aligned}
$$

and $B=1.84$

(Markham and Barker, 1986). Using the mean (unenhanced) DN valuc of the image of 91.85, the calculated spectral albedo is 0.81. Using this value, Equation (12) yields $A=331$. Atmospheric scattering, represented by $T$, is due to three main atmospheric constituents: aterosols, water vapor and particulates (Tanré and others, 1990).
All of these are minor in the Antarctic and we assume here that $T=0$. Substituting these values into Equation (11) and accounting for the image enhancements discussed above and expressed by Equation (14),

$$
\begin{aligned}
A & =3647 \\
\text { and } B & =870 .
\end{aligned}
$$

An alternative, empirical approach to determining $A$ and $B$ is to use the field data to find a best fit for Equation (11). The image data were divided into short segments each beginning and ending at a radar flight line. Any pixel belonged to only one such segment. Each segment was characterized by the mean slope between cnd points calculated from the radar data and the average $D N^{\prime \prime}$ value for all pixels on the segment. Because of the $\pm 3.7 \mathrm{~m}$ standard error in radar elevations, the error in surface slope varies inversely with the segment length and is equal to $\pm 0.18 / N$ where $N$ is the length of the segment in pixels. Segments shorter than 75 pixels $(2137.5 \mathrm{~m})$ were omittcd, lcaving 29471 pairs of slope (expressed as the cosine of the average illumination angle) and avcrage $\mathrm{DN}^{\prime}$. Figure 4 presents this distribution as an intensity histogram. The spread of this distribution in the $\cos \theta$ dimension is approximately 0.0025 , consistent with the 75 pixel cut-off in profile length ( $N=75$ corresponds to a surface-slope error of $\pm 0.0024 \mathrm{rad}$ ).

From Equation (11), $A$ and $B$ represent the slope and intcrcept of the line through this distribution. Although the distribution is highly clustered, the large number of points makes the uncertainty in the determination of $A$ and $B$ very small. Lsing the reduced major-axis method (Davis, 1986), $A=4942.66 \pm 21.41$ and $B=-1212.55 \pm$ 841.0. These values are somewhat different from the values derived above directly from sensor parameters. The difference could be an error in the spectral albedo uscd, the presence of non-negligible atmospheric scattering or even a deviation from the assumed Lambertian reflectance characteristics of the snow surface. These 


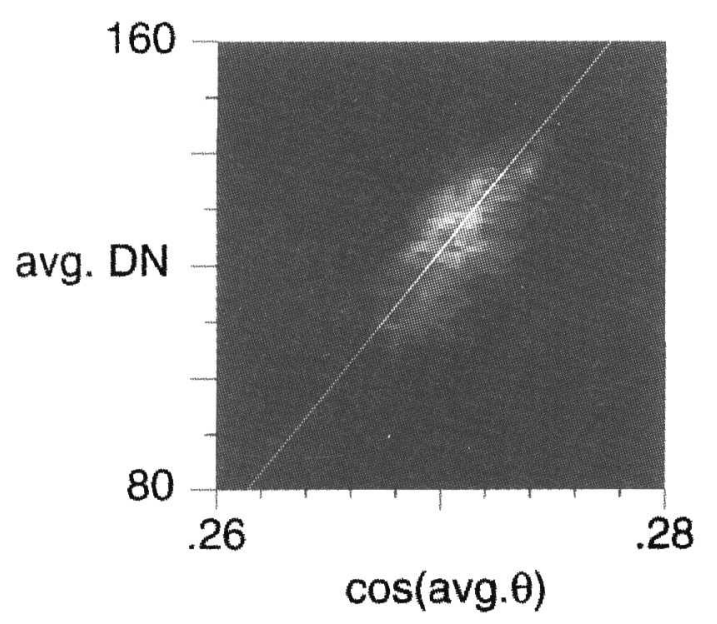

0 256

Fig. 4. Intensity histogram of the paired c'alues of cos (average $\theta$ ) and average $\mathrm{DN}^{-1}$ for all profile segments in the study area longer than 75 pixels. Numbers of points for pach histogram bin are indicated by gray-scale intensity. Least-squares filled line has a correlation coefficient, $r=0.67$.

errors will be discussed in the next section. Nevertheless, because this approach was customized to the actual data, these values of $A$ and $B$ are used below in the application of the method.

Where the method is applied, an clevation is returned for each pixel yielding a digital-elevation model (DFM) with a spatial resolution of $28.5 \mathrm{~m}$. Figure 5 shows the contour-map representation of the DEM resulting from the application of the method to the largest box fully circumscribed by the radar data: $84 \mathrm{~km} \times 84 \mathrm{~km}$ (see Fig. 2). The integration of each profile ran the full length of this large box, beginning at the flight line at the up-Sun boundary and integrating to the down-Sun flight line.
The only correction applied was the cross-Sun smoothing described in a previous section. Figure 5 includes a shaded relief version of this DEM for comparison with the original image. While the brightness values in the shaded relicf image were calculated using the total slope, the DEM was calculated from the original image by assuming image brightness was due solely to the along-Sun component of the surfacc slope. The striking similarity of these two images helps verify that the interpretation of Equation (10), justifying the neglect of cross-Sun slope in our along-Sun integration, is correct.

Comparison of the two contour maps (Figs 3 and 5) illustrates many of the same broad-scale features but also highlights differences. The radar-only DEM is much rougher and contains many isolated peaks and depressions. The image-interpolated DEM was able to use the image data to make appropriate connections between many of the small-scale features left isolated in the radaronly DEM. This is very apparent in the casc of the subtle flow traces that are present in the photoclinometric DEM but absent in the radar-only map. The image-interpolated DEM appears much smoother yet retains the smallscale topographic structure.

Each integration profile crossed a number of intervening flight lines for which elevations could be compared. The net residual for these 41230 crossing points was $7.46 \pm 11.73 \mathrm{~m}$. The mean is within one standard deviation of zero. Figure 6 is a contour map of clevation residual contoured from the calculated residuals (radar-only minus photoclinometric) along the flight lines. The contour intervals are shaded above $5 \mathrm{~m}$ and below $-2 \mathrm{~m}$ values that correspond roughly to $1 \sigma$ above and $1 \sigma$ below the mean crossing-point residual. Most of the pixcls exceeding the $l \sigma$ level are spatially coherent. The region where the image-interpolated elevations are above the radar-only elevations occurs in one of the roughest topographic regions. Image-interpolated clevations above the radar-only elevations are limited mainly to the region left of center, where little topographic variation is evident.
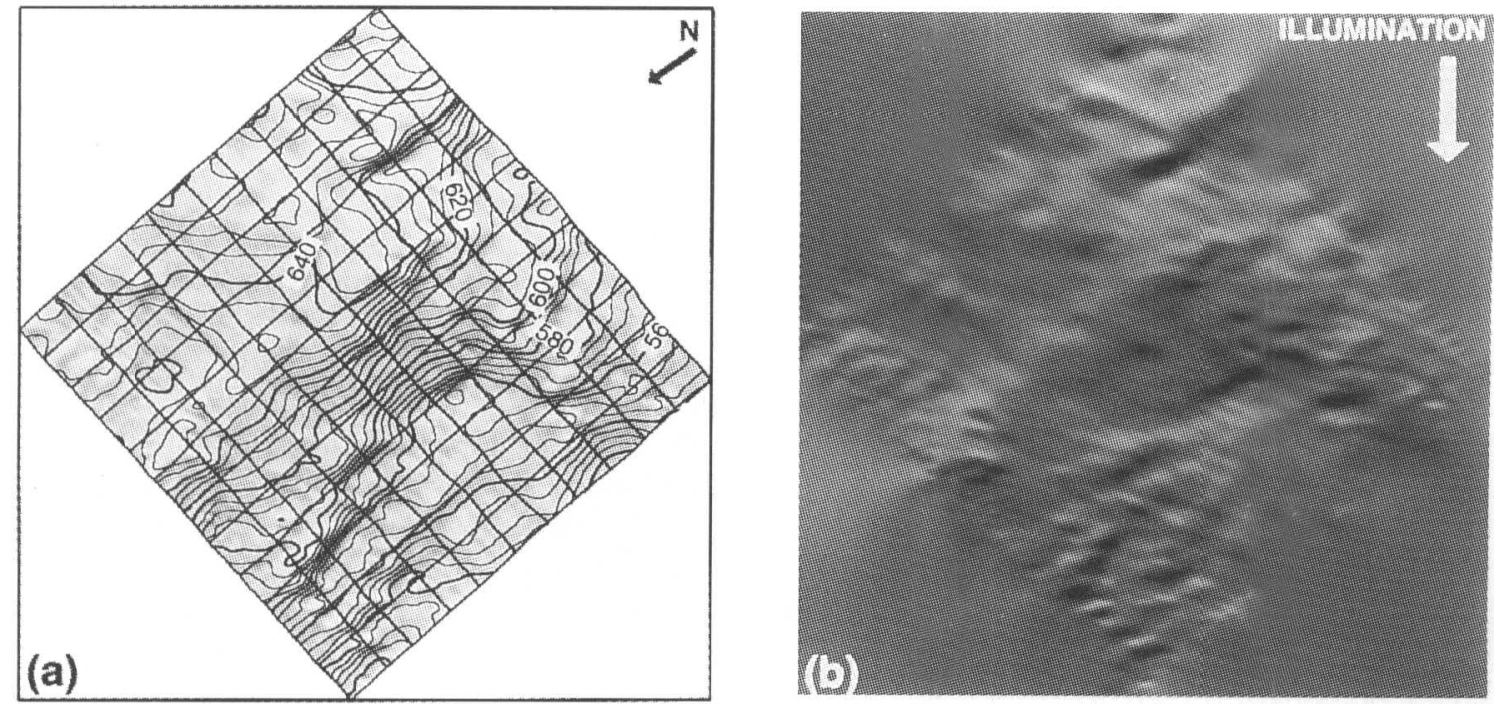

Fig. 5. a. Elezalion-contour map based on image interpolation of airborne-radar data using Equalion (11). Contour interval is $5 \mathrm{~m}$. Contours are superimposed on original image. $b$. Shaded relief zersion of contours in (a) illuminated from the same direction as the original image. 


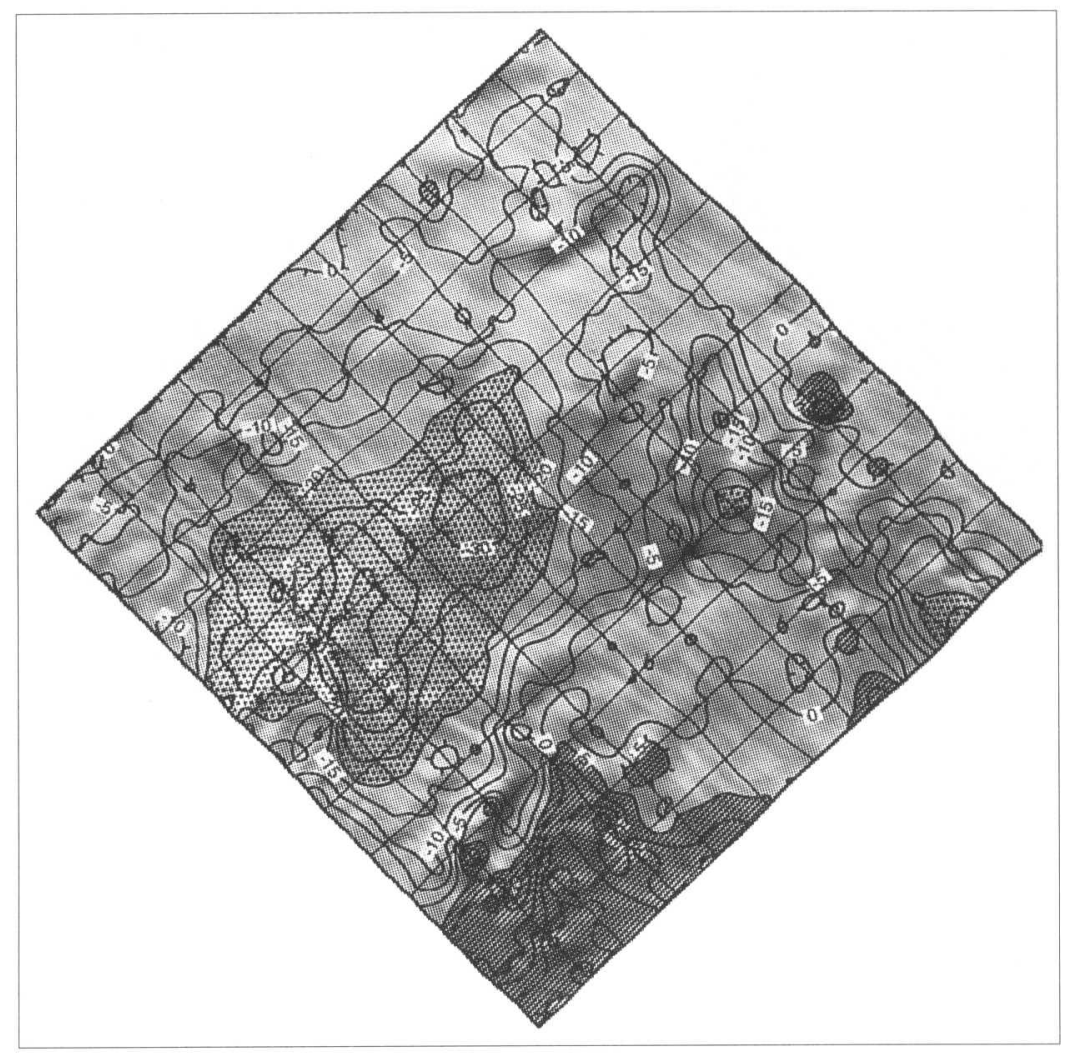

Fig. 6. Contour plot of elevation residual (radar minus photoclinometric). Shaded areas are less than $-20 \mathrm{~m}$ and greater than $5 \mathrm{~m}-$ approximately $1 \sigma$ limits from the mean residual value.

The above test case was the extreme where only the flight line farthest up-Sun served as control while all others were used to check accuracy. By using more flight lines for control, the integration distance can be shortencd and the effect of integration distance on residuals can be examined. This study was conducted for a suite of cases, each with a differcnt integration distance corresponding to some integral number of flight-line spacings. As an example, in the shortest integration-distance case, every north-south-oriented flight line was used to begin an integration that proceeded through the next flight line down-Sun and ended at the sulsecquent flight line. Each along-Sun profile was adjusted linearly so the elevations matched at the up-Sun and down-Sun boundarics. Residuals were then calculated at all points along intervening flight lines.

The results of this study are illustrated in Figure 7 which plots the standard deviation of the residuals versus integration distance. The rise in standard deviation with integration distance is nearly linear. This error is the total error and is a combination of errors in the radar data and errors in the photoclinometric method. Because these two error sources are independent,

$$
\sigma_{t}^{2}=\sigma_{r}^{2}+\sigma_{p}^{2}
$$

where $\sigma_{\mathrm{t}}, \sigma_{\mathrm{r}}$ and $\sigma_{\mathrm{p}}$ refer to the total crror, the radar crror and the photoclinometric-method error, respectively. As can be seen from Figure 7, for integration distances less than $19 \mathrm{~km}$, the photoclinometric method has a smaller error than the radar measurement.

While it is useful to study the overall pattern of difference between the radar and photoclinometric DEMs

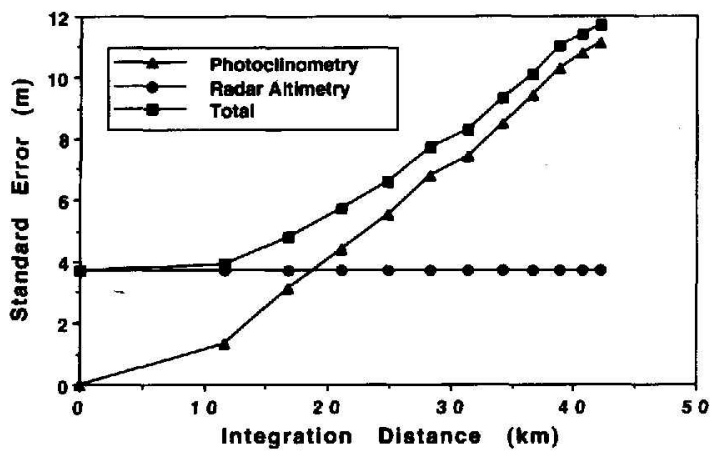

Fig. 7. Elevation errors $(1 \sigma)$ of the radar minus photoclinometric elevation residuals versus integration distance. Total error is treated as the root-summed-square of the radar error (taken from Retzlaff and others, 1993) and photoclinometric error (calculated from Equation (15)).

as was shown in Figure 6, it also is valuable to look at how specific elevation profiles compare. Figure 8 shows two elevation profiles along flight lines indicated in Figure 2. The photoclinometric profiles were calculated using an integration distance of $12 \mathrm{~km}$ - thus, they were controlled $6 \mathrm{~km}$ upstream and $6 \mathrm{~km}$ down-Sun. Profile A-A' shows very good agreement. The disagreement is worst in the middle section where the radar-derived elevations lie beneath the photoclinometric elevations but the majority of the differences are well within one standard deviation of the errors. Profile $B-B^{\prime}$ illustrates a different sort of difference which strongly suggests a $2 \mathrm{~km}$ horizontal crror in the co-registration of the radar and image data. Co- 

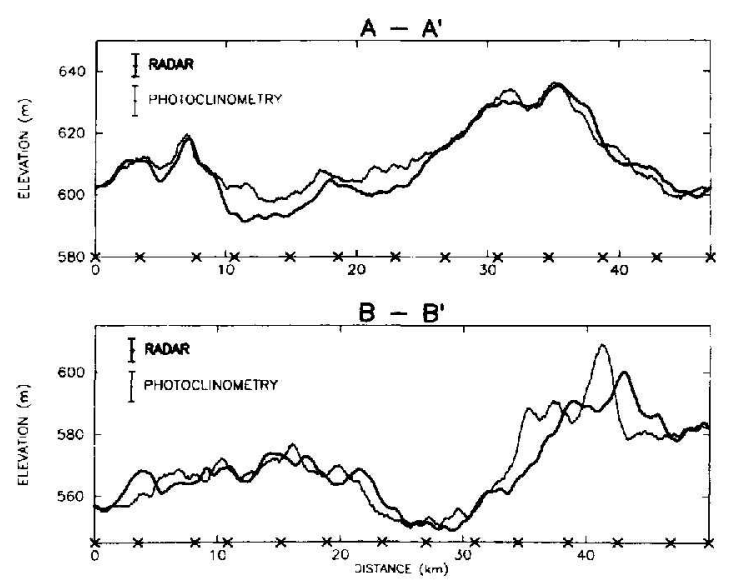

Fig. 8. Elevation profiles comparing radar data (heavy) and photoclinometrically calculated (light) results along two flight lines. Error bars indicate $1 \sigma$ error levels. Location of profiles is indicated in Figure 2. Photoclinometric resulls were calculated using an integration distance of approximately $12 \mathrm{~km}$. "X"s along the horizontal axis represent the intersection of orthogonal flight lines with the profile.

registration accuracy is discussed in the next section.

When the integration was performed using the theoretical values of $A$ and $B$ discussed in the beginning of this section, the resulting DEM was similar but included a mean slope that was not present using the empirical values. This additional slope was the result of Equation (11) defining a line in Figure 4 that did not pass through the mean of the distribution. When the value of $B$ was modified to pass through the distribution mean, a very similar DFM and a residuals map similar to Figure 6 resulted. The DEM and the corresponding residuals map proved to be rather insensitive to the variations in $A$ and $B$ as long as Equation (11) satisfied the mean values of $\mathrm{DN}^{\prime}$ and $\cos \theta: 124.78$ and 0.2706 , respectively.

An interesting use of the DEM is to illuminate it along and transverse to the general direction of motion. Ice
Stream $\mathrm{C}$ is now virtually stagnant; however, the features still remain from when it was active and are typical, albeit subdued, of active ice streams. The different illuminations in Figure 9 show a clcar anisotropy in the surface topography. Viewed along-flow, the longer-scale roughness of the surface is apparent. We feel this perspective represents a qualitiative display of where the ice flow over the bed is either encumbered (rough topography) or unencumbered (smooth surfacc). It may correlate well with basal topography (a study soon to be undertaken). The cross-flow view highlights the elongated nature of the surface topography. This elongation is presumably a result of flow (paleo-flow in this case). Ridges clcarly cvident in this perspective all but disappear in the along-llow view.

\section{ERROR SOURGES}

Many of the error sources have already been discussed. Those remaining include co-registration of the data sets, noise in the data and violations of the assumptions of constant albedo and Lambertian reflectance. These are discussed below.

Accurate co-registration depends on adequate positioning of both the aircraft as it collected radar data and the satellite as it collected the image data. Retzlaff and others (1993) have stated that the misclosure of the aircraft-navigation system for their flights had a mean of $2 \mathrm{~km}$ with a standard deviation of nearly $1 \mathrm{~km}$. This error is included in their $\pm 3.7 \mathrm{~m}$ figure for the overall error. Image location has been determined from an overall gcorefercnced registration of $16 \mathrm{TM}$ images carried out by the L.S.G.S. and is accurate to $\pm 500 \mathrm{~m}$ (Ferrigno and others, 1994). Elevation errors resulting from either misregistration or inaccuracies in the Sun's position are very small and would tend to be removed by the empirical approach taken to determine $A$ and $B$. They might, however, contributc to the difference between the empirical values and the theoretical values of $A$ and $B$.

$\mathrm{DN}$ values generally are accurate to $\pm 1 \mathrm{DN}$ (personal communication from B. L. Markham). Given the narrow
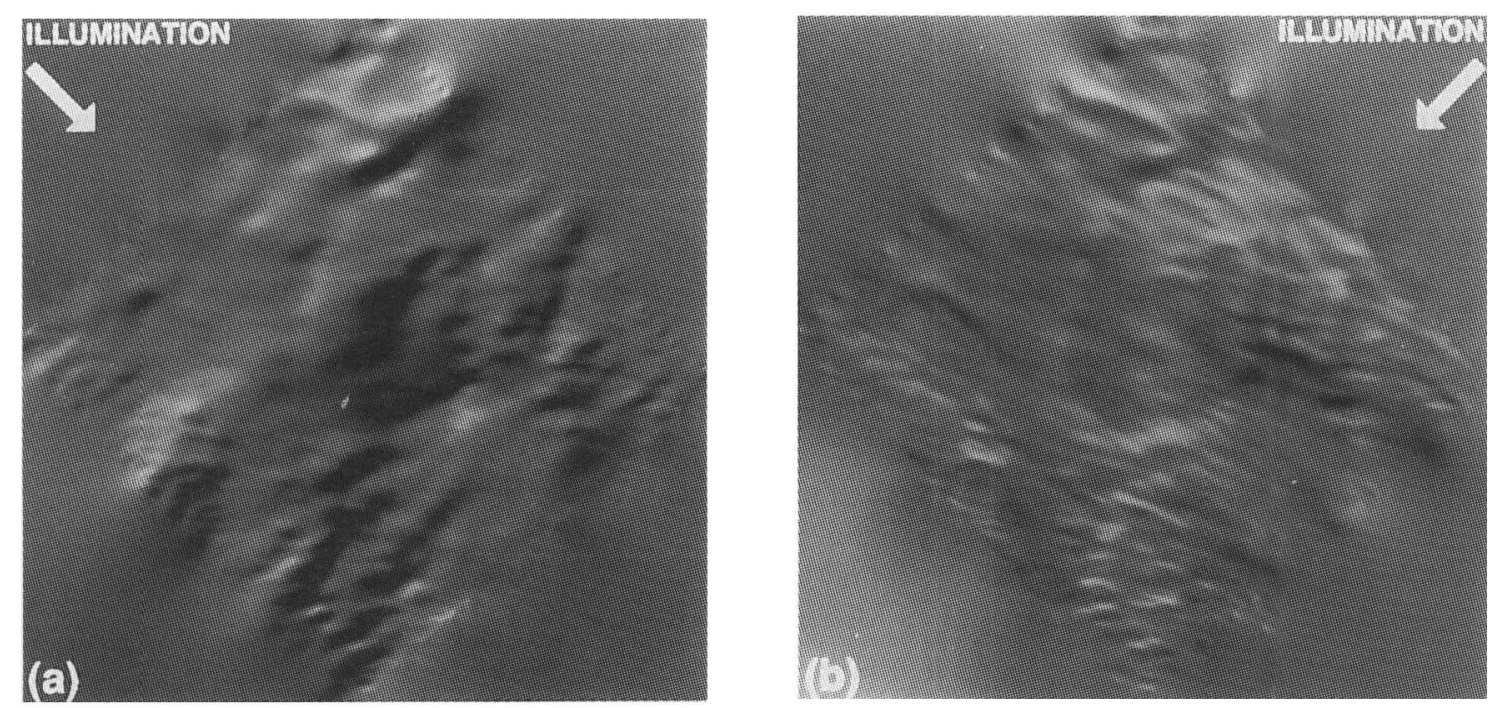

Fig. 9. Shaded relief images of the photoclinometric elevation field illuminated (a) along-flow and (b) cross-flow, as indicated. 
range of $\mathrm{DN}$ values for this image $\angle 12 \mathrm{DN}$ for the original image), this represents a substantial fraction of the full range of brightness across the image. Equation (14) shows that this uncertainty was expanded to $\pm 11 \mathrm{DN}^{\prime}$ after image enhancement. The $5 \times 5$ pixel running average should reduce this error by a factor of 5 , if this error is truly random. However, there is a non-random component, as evidenced by stripes in the enhanced image. Algorithms usually can remove completely periodic components, such as scan-line noise (as was done to this image) but other components must be targeted individually by visual inspection. The expression in the derived DEM of unidentified noise of this type would be a ramp extending across the entire image in the residual field. -Since no such artifacts appeared, this noise source probably was dealt with adequately. The spread of the points in the $\mathrm{DN}^{\prime}$ dircction in the intensity histogram (Fig. 4) provides an empirical measure of the uncertainty in $\mathrm{DN}^{\prime}$. From this plot, the approximate $1 \sigma$ uncertainty is $\pm 5 \mathrm{DN}^{\prime}$. This converts to an effective uncertainty of only $\pm 0.5 \mathrm{DN}$ in the original image, in rough agreement with the expected accuracy quoted by Markham.

The assumption of Lambertian reflectance led to the cosine relationship between image brightness and illumination angle. The disagreement between the empirical and theoretical values of $\beta$ and $B$ suggests that this assumption may not be valid. Curiously, careful field measurements of the bi-directional reflectance function cover many geometries but stop short of the nadir-viewing case (Steffen, 1987). In our case, the surface rclief is too small to use adequately the distribution of data in Figure 4 to test whether the Lambertian assumption is valid. In practice, a linear relationship such as Equation (11) works reasonably well over a limited rangc of slopes.

Finally, in Equation (11) we assumed that the spectral albedo was constant, thus attributing all DN variations to topographic variations. In general, this is not truc. Regions of different albedo are seen in ice-sheet imagery with easily identifiable sharp boundaries, even though the DN contrast across the boundary is only 1 or $2 \mathrm{DN}$. No such sharp boundaries existed in this study region but that does not eliminate the possibility that more gradual albedo variations are present. The effect of an undetected area of different albedo is to gencrate a ramp in the derived elcvation field. The amount of albedo variation required to create an apparent slope increment can be derived from Equation (11) as

$$
\frac{\partial R}{\partial \theta}=R \tan \theta
$$

For our case, $\theta$ and $R$ are approximately 748 and 0.86 , respectively. Thus, a $1 \%$ change in $R$ is equivalent to a change of $0.003 \mathrm{rad}$ in $\theta$, or $0.095 \mathrm{~m} \mathrm{pixel}^{-1}$. To attribute the region of highest residual (seen in the left half of Figure 6) to this effect, the $35 \mathrm{~m}$ rise over $15 \mathrm{~km}$ requires only a $0.7 \%$ increase in albedo. This would correspond to a similar percentage change in DN. As mentioned earlier, the mean DN for the original, unenhanced scene was 91.5 so this percentage change amounts to only $0.64 \mathrm{DN}$. It is certainly possible that an albedo change of this magnitude would go undetected. Thus, albedo variations appear to be the most likely source of the larger errors in the photoclinometric method occurring at larger integration distances.

\section{CONCLUSIONS}

We conclude that photoclinometric techniques can provide useful topographic information on ice sheets. The insensitivity of the image brightness to the cross-Sun slope can be used to advantage by integrating surface slopes derived from image brightness along lines parallel to the Sun's azimuth. A requirement of such an integration is independent knowledge of at least one elevation for each line. Additional elevation control can improve the accuracy of the application by permitting an empirical fit of the data to obtain values for the two required parameters ( $A$ and $B$ ) in Equation (11). Because errors in the calculated clevations increase with integration distance, the more frequently spaced the control, the more accurate the photoclinometric DEM.

The major limitations of the method are all tied to the radiance resolution of the Landsat TM sensor. Ice-sheet surface slopes are small and narrow histograms of image brightness amount to a condition of small signal-to-noise ratio. An additional complication is that subtle variations in albedo can remain undetected under such conditions. I arger contrasts in albedo exhibit sharp boundaries that suggest the possibility of identification of less-extreme albedo contrasts if the sensor-radiance resolution is increased. Finally, lower Sun-elevation conditions cannot substitute for improved radiance resolution. The sensitivity of the illumination angle to surface slope is already at $96 \%$ of its maximum value at a solar elevation of $16^{\circ}$.

This study was done as a result of a fortuitous coincidence of the required data sets. Our test data were far from ideal. Errors in the radar-measured surface elevations were clearly evident in some cases and limited the ability to define clearly the source of the residual errors. A better-controlled collection of data specifically designed to study the potential of photoclinometry would likely remove some of the ambiguity remaining from this study.

\section{ACKNOWLEDGEMENTS}

We gratefully acknowledge C.R. Bentley for making available to us the University of Wisconsin airborne-radar data. N. Lord gave us additional information on the collection and characteristics of these data. Throughout the course of this research, M. Fahnestock offered many useful suggestions and helped in the preparation of the figures. Two anonymous reviewers and D. Wingham also made constructive comments that improved the text. This research was supported by the U.S. National Science Foundation under grant DPP-9018127.

\section{REFERENCES}

Cooper, A.P.R., N.F. McIntyre and G.de Q. Robin. 1982. 1)riving stresses in the Antarctic ice sheet. Arm. Glaciol., 3, 59-64.

Crippen, R. E. 1989. A simple filtering routine for the cosmetic removal of scan-line noise from Landsat TM P-tape imagcry. Photogramm. Eng. Remote Sensing, 55(3), 327-331. 
Davis, J. C. 1986. Statistics and data analysis in geology. Second edition. New York, John Wiley and Sons.

Dowdeswcll, J. A, and N. F. McIntyre. 1987. The surface topography of large ice masses from Landsat imagery. F. Glaciol., 33(113), 16-23.

Ferrigno, J. G. and 7 others. 1994. Landsat TM image maps of the Shirase and Siple Coast icc streams, West Antarctica. Ann. Glaciol., 20 (see paper in this volume).

Markham, B.L. and J. L. Barker. 1986. Landsat MSS and TM postcalibration dynamic ranges, exoatmospheric reflectances and atsatellite temperatures. EOSAT Terhical Notes 1, 3-8.

Rees, W.G. and J.A. Dowdeswell. 1988. Topographic eflects on light scattering from snow. Proc. IGARSS'88 Symp. ESA SP-284, 161164.

Retaliff, R., N. Lord and C. R. Bentley. 1993. Airborne radar studies: Ice Streams A, B and C, West Antarctica. 7. Glaciol, 39(133), 495-506.

Steffen, K. 1987. Bi-directional reflectance of snow at 500-600 nm. International Association of Hydrological Sciences Publication 166 (Sym- posium at Vancouver 1987 Large Scale Effects of Seasonal Snow Cover), 415-425.

Tanrc, D. and 6 others. 1990. Description of a computer code to simulate the satellite signal in the solar spectrum: the $5 \mathrm{~S}$ code. Int. 7 . Remote Sensing, 11, 659.668.

Vaughan, D.G., C.S.M. Doake and D.R. Mantripp. 1988. I'opography of an Antarctic ice stream. In SPOT-1 image utilization. assessment, results. Toulouse, CNES Cepadues Editions, 167-174.

Wildey, R. L. 197j. Generalized photoclinometry for Mariner 9. Icarus, 25, 613-626.

The accuracy of references in the text and in this list is the responsibility of the authors, to whom queries should be addressed. 